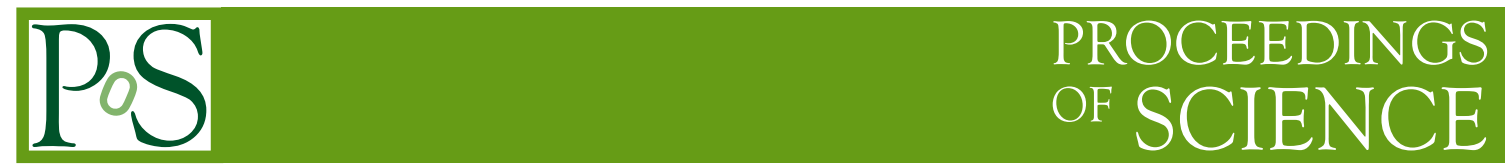

\title{
Low Mass Higgs at the Tevatron
}

\author{
Satish Desai* ${ }^{\dagger}$ \\ Fermi National Accelerator Laboratory \\ E-mail: satish@fnal.gov
}

We report on recent searches for the production of a low mass standard model Higgs boson conducted at the Tevatron $p \bar{p}$ collider. The most promising channels at Higgs masses less than $~ 135$ $\mathrm{GeV}$ are for the production of a Higgs decaying to $b \bar{b}$ or $\tau \tau$ in association with a $W$ or $Z$ boson decaying leptonically. Both the CDF and D0 collaborations have conducted searches in $2.0 \mathrm{fb}^{-1}$ $5.2 \mathrm{fb}^{-1}$ of data collected at $\sqrt{s}=1.96 \mathrm{TeV}$. No evidence for a Higgs boson is seen. Therefore the results from the two experiments are combined to set $95 \%$ confidence level limits on the Higgs cross section. At a Higgs mass of $115 \mathrm{GeV}$, the observed (expected) limit is 2.70 (1.78) times the standard model cross section.

XXth Hadron Collider Physics Symposium

November 16 - 20, 2009

Evian, France

* Speaker.

$\dagger$ The author would like to thank the D0 and CDF collaborations for the efforts that contributed to these proceedings. Thanks are also due to the Fermilab accelerator division for delivering the large datasets presented here. Finally the author would like to thank the HCP organizers for an excellent conference with many fascinating presentations. 


\section{Introduction}

The nature of electroweak symmetry breaking is one of the most important unresolved issues in particle physics. The standard model (SM) addresses this problem through Higgs mechanism. In addition to generating masses for the $W$ and $Z$ bosons, the model predicts a heavy spin 0 particle, the Higgs boson $(H)$. In these proceedings, we present the latest results in the search for a SM Higgs boson at the Tevatron collider.

Higgs bosons are produced at the Tevatron primarily by gluon fusion through a top quark loop: $g g \rightarrow H$. However, at masses below $\sim 135 \mathrm{GeV}$, the decay $H \rightarrow b \bar{b}$ dominates, and the large multijet backgrounds make a search in this channel impractical. Therefore searches for a light Higgs boson focus on the production of a Higgs boson in association with a $W$ or $Z$ boson.

Here we discuss the searches for the $Z H \rightarrow v v b \bar{b}, W H \rightarrow l v b \bar{b}$, and $Z H \rightarrow l l b \bar{b}$ processes. We also discuss searches in the $\tau \tau j j$ topology, which receives contributions from several Higgs production modes.

\section{Searches in the $\mathbb{t}_{T}+b \bar{b}$ Channel}

Both CDF and D0 search for a Higgs in events containing two $b$-jets and a large missing transverse energy $\left(\mathbb{E}_{T}\right)$ [1], [2]. This final state is sensitive to $Z H \rightarrow v v b \bar{b}$ production, as well as the $W H \rightarrow l v b \bar{b}$ process, when the charged lepton is not reconstructed. The D0 result is newly updated for this conference.

After the initial selection of events with high $\mathbb{E}_{T}$ and at least two jets, the background is dominated by multijet processes in which the $\mathbb{E}_{T}$ arises from mismeasurement of the jet energies. Other backgrounds processes include $W$ or $Z$ plus jets, $t \bar{t}$ and diboson production. These backgrounds typically contain charged leptons, so events containing an electron or muon are rejected. This also ensures that the $\mathbb{E}_{T}+b \bar{b}$ searches are orthogonal the searches that require the presence of an electron or muon.

Both collaborations model the multijet background with control samples in collider data. In the case of D0, the control sample is derived by requiring that the $\mathbb{E}_{T}$ as measured in the calorimeter is separated in azimuth from the measurement obtained using charged tracks. The search is conducted using events in which the two measurements are well aligned. Other backgrounds are estimated using Monte Carlo simulations that are validated in events that contain a muon but with otherwise identical selection requirements.

At least one of the selected jets must be identified ( $b$-tagged) as containing a $b$-quark. CDF uses two taggers, one based on the reconstruction secondary vertices within the jet, and one that uses high impact parameter tracks associated with the jet. D0 has a single neural net (NN) $b$-tagger that makes use of both secondary vertex and track impact parameter information. To maximize sensitivity, both experiments consider events with one or two tagged jets separately, using more stringent requirements for events with only one tagged jet.

Backgrounds are further suppressed through use of multivariate discriminants. D0 uses decision trees (DTs), whereas the CDF analysis makes use of NNs. Each experiment trains two discriminants, one against the multijet events, and one against the remaining (electroweak) backgrounds. Selection requirements are placed on the multijet discriminants, and limits are extracted 

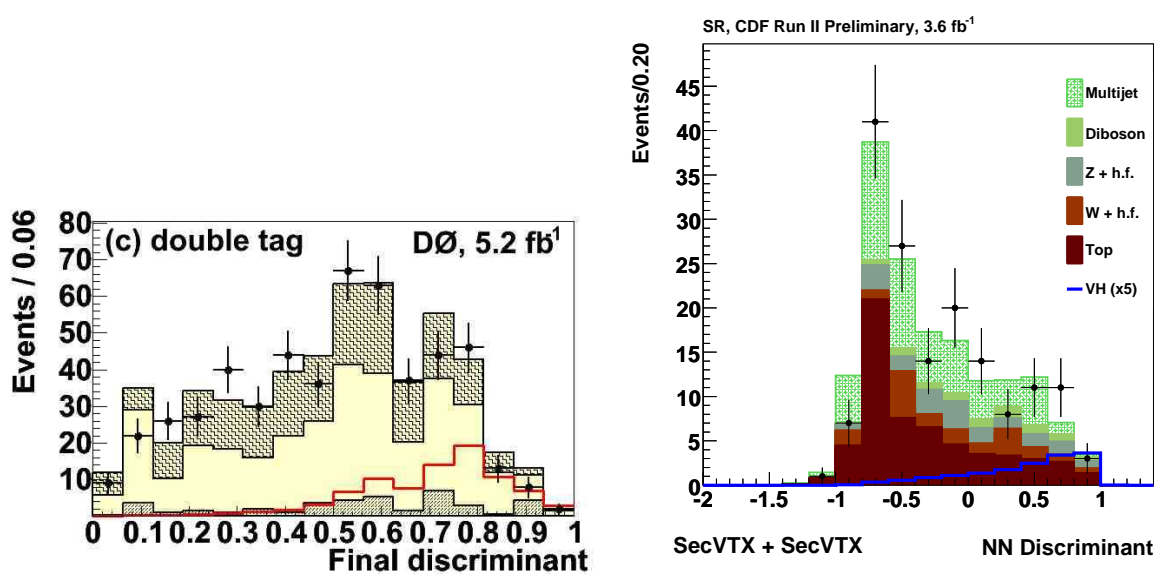

Figure 1: Final discriminants for the $\mathbb{E}_{T}+b \bar{b}$ search in events with two $b$-tagged jets from D0 (left) and $\mathrm{CDF}$ (right). A Higgs mass of $115 \mathrm{GeV}$ is assumed for these distributions.

from the shape of the electroweak background discriminants, shown in Fig. 1. At 95\% confidence level, D0 excludes a Higgs with a cross section 3.7 times that of the SM cross section $\left(\sigma_{S M}\right)$ at a Higgs mass of $115 \mathrm{GeV}$. The corresponding limit from CDF is $6.1 \sigma_{S M}$.

\section{Searches in the $W H \rightarrow l v b \bar{b}$ Channel}

In this section we present searches from CDF and D0 for $W H \rightarrow l v b \bar{b}$ with $l=e, \mu$ [3], [4]. $\mathrm{CDF}$ has a new result for this conference using a final discriminant based on matrix elements. Additionally, we present a new result from D0 that searches in the $\tau v b \bar{b}$ final state [5]

Both of the $l v b \bar{b}$ analyses select events containing a high $p_{T}$ electron or muon, large $\mathbb{E}_{T}$ and two or three jets. The $\tau v b \bar{b}$ search from D0 requires a one prong hadronic tau instead of an electron or muon, and at least two jets. Additionally, all analyses demand that least one jet is $b$-tagged. As in the $\mathbb{E}_{T}+b \bar{b}$ searches, events are split into orthogonal samples based on the number of jets and the number and quality of $b$-tags, and limits are extracted by considering each sample independently.

After initial selection, CDF evaluates a matrix element discriminant (also called the event probability discriminant) to separate the $W H$ signal from the backgrounds, which include contributions from $W+$ jets (including heavy flavor jets), , single top and diboson events. Transfer functions are used to connect experimental observables to the differential cross sections for the process considered in the discriminant. Neural nets improve the transfer functions for the jet energies by incorporating additional information such as the momenta of tracks associated with each jet.

In the D0 $l v b \bar{b}$ analysis neural nets are used to extract the signal, while the $\tau v b \bar{b}$ search uses boosted decision trees (BDTs). Distributions of the final discriminants are shown in Fig. 2. CDF observes a limit of $6.6 \sigma_{S M}$ rate in the matrix element search. However, the combination presented in Section 6 uses only three jet events from this analysis. The two jet sample is obtained from an older neural net based analysis. The D0 $l v b \bar{b}$ and $\tau v b \bar{b}$ limits are $6.9 \sigma_{S M}$ and $14.1 \sigma_{S M}$ for a 115 GeV Higgs, respectively. The D0 $\tau v b \bar{b}$ is not used in the combination, because the selection is not yet orthogonal to that of the D0 $\mathbb{E}_{T}+b \bar{b}$ search. 

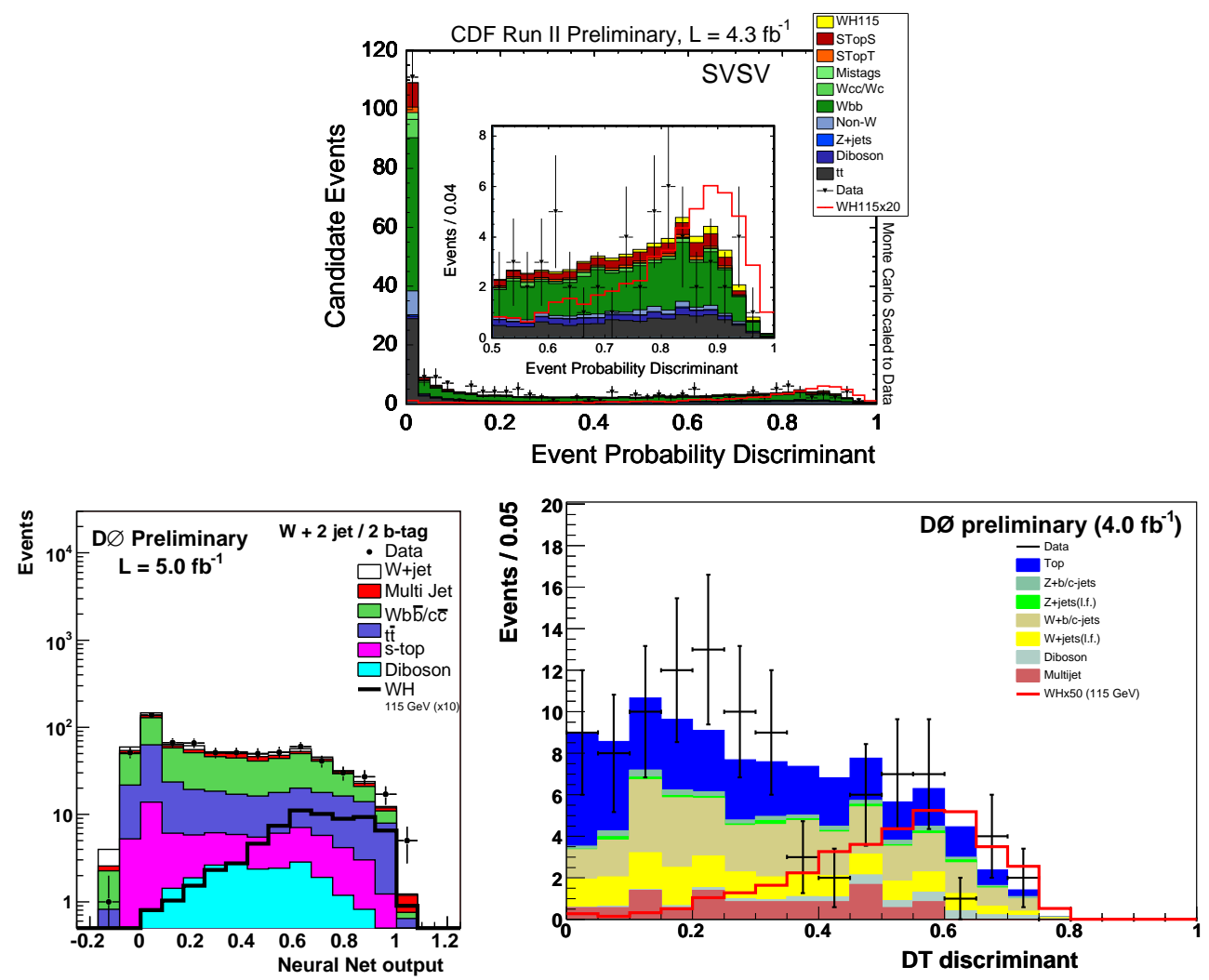

Figure 2: Final discriminants for the $l v b \bar{b}$ search in events with two $b$-tagged jets from CDF (top), the D0 $l v b \bar{b}$ search (bottom left) and the D0 $\tau v b \bar{b}$ search (bottom right), assuming a Higgs mass of $115 \mathrm{GeV}$. An expanded view of the high signal region is shown for the CDF distribution.

\section{Searches in the $Z H \rightarrow l l b \bar{b}$ Channel}

The $l l b \bar{b}$ searches by D0 and CDF both take advantage of the low backgrounds and fully reconstructed final state [6], [7]. Events are required to have two electrons or muons and at least two jets. Both experiments utilize specialized lepton identification criteria to recover gaps in the acceptance for electrons and muons. As in other searches with $H \rightarrow b \bar{b}$ at least one $b$-tag is required. Additionally, the reconstruction of the Higgs mass resonance is improved by the use of a kinematic fit from D0, and a NN based jet energy correction from CDF.

CDF extracts limits from a two dimensional $\mathrm{NN}$ trained against and $Z+$ jets backgrounds. D0 uses a one dimensional BDT. Distributions of these discriminants are shown in Fig. 3. For the CDF NN, only a one dimensional slice in the region with low $t \bar{t}$ contribution is displayed. For a $115 \mathrm{GeV}$ Higgs, CDF observes a limit of $5.9 \sigma_{S M}$, compared to a limit of $9.1 \sigma_{S M}$ from D0.

\section{Searches in the $\tau \tau j j$ Final State}

There are several Higgs production mechanisms that contribute to the $\tau \tau j j$ final state. Because of the $\sim 10 \%$ branching fraction for $H \rightarrow \tau \tau$, the $Z H$ process contributes events with both $H \rightarrow b \bar{b}$ 

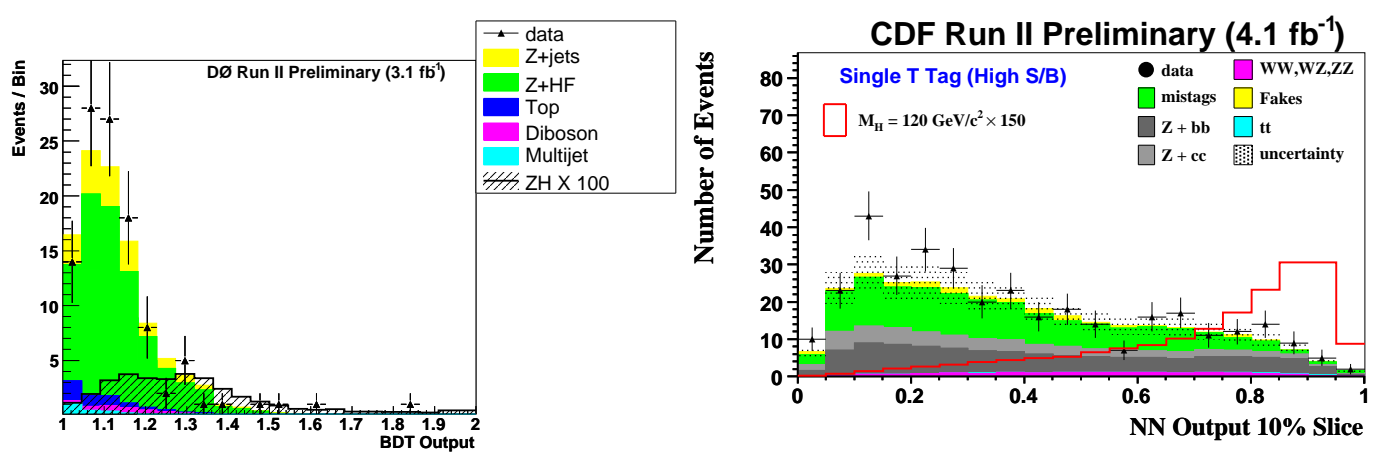

Figure 3: Final discriminants for the $l l b \bar{b}$ search from D0 (left) and CDF (right) in events with only one tagged jet. A Higgs mass of $115 \mathrm{GeV}$ is assumed for these distributions.
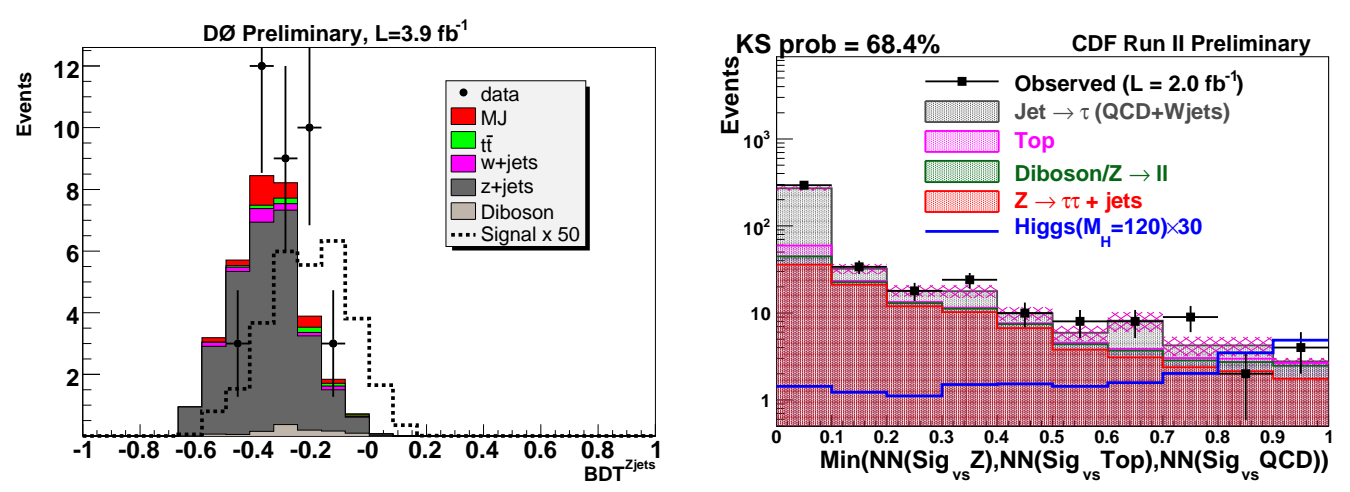

Figure 4: Final discriminants for the $\tau \tau j j$ search froom D0 (left) and CDF (right).

and $H \rightarrow \tau \tau$ decays. Additionally, gluon fusion and vector boson fusion Higgs production also contribute significantly.

The event selection requires two jets and one hadronic $\tau$ and one muon. CDF additionally accepts events with an electron instead of a muon. Because of the multiple signal processes, several multivariate discriminants are trained to extract Higgs events. The discriminants are then combined and limits extracted. Distributions of the final discriminants may be seen in Fig. 4. CDF observes a limit of $25 \sigma_{S M}$ at a Higgs mass of $115 \mathrm{GeV}$, while the limit of from D0 is $16 \sigma_{S M}$.

\section{The Tevatron Combination}

No one search channel is sensitive to a Higgs produced at the rate predicted by the SM. Therefore it is necessary to combine the results from all searches. The CDF and D0 collaborations have released a new combination for this conference [10]. The combination includes the results presented here as well as those from other searches. The expected and observed limits, expressed as a ratio of the SM cross section, and as a function of assumed Higgs mass are shown in Fig. 5. At a mass of $115 \mathrm{GeV}$, a 95\% confidence level upper limit of 2.70 (1.78) times the SM Higgs cross section is observed (expected). 


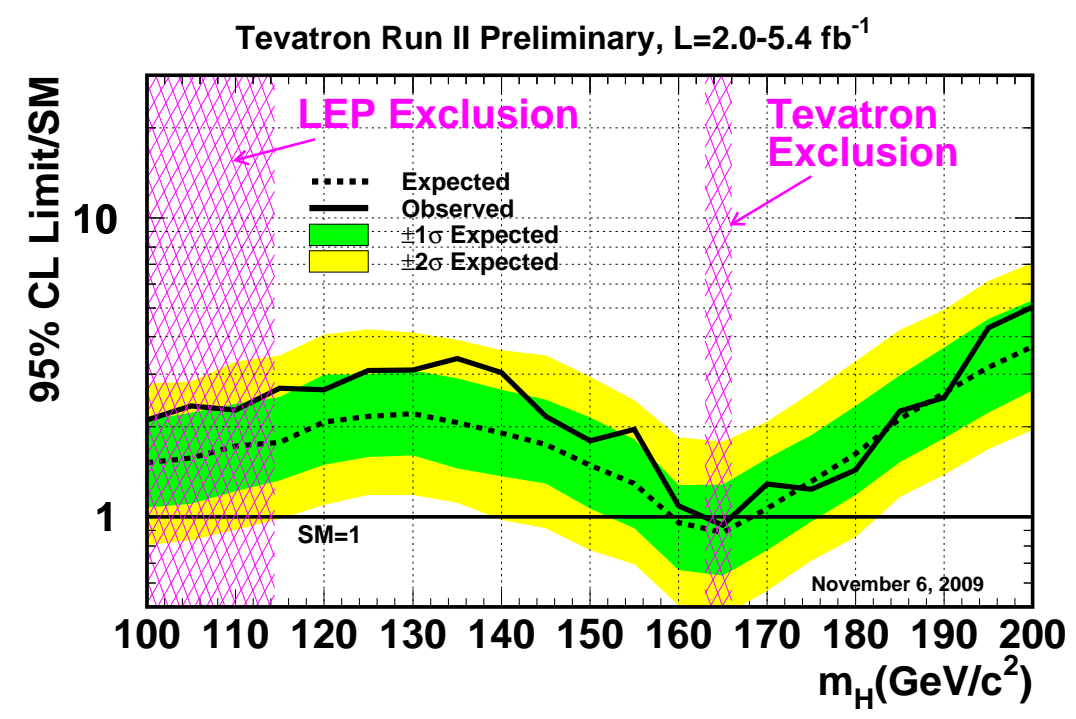

Figure 5: Expected (dashed line) and observed (solid line) 95\% confidence level limits as divided by the SM cross section as a function of assumed Higgs mass. The green and yellow bands indicate the $1 \sigma$ and $2 \sigma$ variations on the expectated limit.

\section{Summary}

We have presented the results of recent searches for a low mass Higgs boson at the D0 and $\mathrm{CDF}$ experiments. Searches were conducted on datasets ranging from $2.0 \mathrm{fb}^{-1}$ to $5.2 \mathrm{fb}^{-1}$. After combining the results of the searches from both experiments, a limit of 2.70 times the standard model cross section was observed, for a Higgs with a mass of $115 \mathrm{GeV}$.

\section{References}

[1] The CDF Collaboration, CDF Note 9642

[2] V.M. Abazov et al. [The D0 Collaboration], arXiv:0912.5285 [hep-ex]

[3] The CDF Collaboration, CDF Note 9985

[4] The D0 Collaboration, D0 Note 5972-CONF

[5] The D0 Collaboration D0 Note 5977-CONF

[6] The D0 Collaboration, D0 Note 5876-CONF

[7] The CDF Collaboration, CDF Note 9889

[8] The D0 Collaboration, D0 Note 5845

[9] The CDF Collaboration, CDF Note 9248

[10] Tevatron New Phenomena \& Higgs Working Group, arXiv:0911.3930 [hep-ex] 